\title{
Furfural tolerance and detoxification mechanism in Candida tropicalis
}

\author{
Shizeng Wang ${ }^{1}$, Gang Cheng ${ }^{1}$, Chijioke Joshua² ${ }^{2}$ Zijun He${ }^{1}$, Xinxiao Sun ${ }^{1}$, Ruimin Li ${ }^{1}$ Lexuan Liu ${ }^{1}$ \\ and Qipeng Yuan ${ }^{1 *}$
}

\begin{abstract}
Background: Current biomass pretreatment by hydrothermal treatment (including acid hydrolysis, steam explosion, and high-temperature steaming) and ionic liquids generally generate inhibitors to the following fermentation process. Furfural is one of the typical inhibitors generated in hydrothermal treatment of biomass. Furfural could inhibit cell growth rate and decrease biofuel productivity of microbes. Candida tropicalis is a promising microbe for the production of biofuels and value-added chemicals using hemicellulose hydrolysate as carbon source. In this study, $C$. tropicalis showed a comparable ability of furfural tolerance during fermentation. We investigated the mechanism of C. tropicalis's robust tolerance to furfural and relevant metabolic responses to obtain more information for metabolic engineering of microbes for efficient lignocellulose fermentation.

Results: Candida tropicalis showed comparable intrinsic tolerance to furfural and a fast rate of furfural detoxification. C. tropicalis's half maximal inhibitory concentration for furfural with xylose as the sole carbon source was $3.69 \mathrm{~g} / \mathrm{L}$, which was higher than that of most wild-type microbes reported in the literature to our knowledge. Even though furfural prolonged the lag phase of $C$. tropicalis, the final biomass in the groups treated with $1 \mathrm{~g} / \mathrm{L}$ furfural was slightly greater than that in the control groups. By real-time PCR analysis, we found that the expression of $A D H 1$ in $C$. tropicalis ( $C$ ADDH1) was induced by furfural and repressed by ethanol after furfural depletion. The expression of $c t A D H 1$ could be regulated by both furfural and ethanol. After the disruption of gene $C t A D H 1$, we found that $C$. tropicalis's furfural tolerance was weakened. To further confirm the function of $\mathrm{CtADH1}$ and enhance Escherichia coli's furfural tolerance, CtADH1 was overexpressed in E. coli BL21 (DE3). The rate of furfural degradation in E. coli BL21 (DE3) with pET-ADH1 (high-copy plasmid) and PCS-ADH1 (medium-copy plasmid) was increased by 1.59-fold and 1.28-fold, respectively.

Conclusions: Candida tropicalis was a robust strain with intrinsic tolerance to inhibitor furfural. The mechanism of furfural detoxification and metabolic responses were identified by multiple analyses. Alcohol dehydrogenase 1 was confirmed to be responsible for furfural detoxification. C. tropicalis showed a complex regulation system during furfural detoxification to minimize adverse effects caused by furfural. Furthermore, the mechanism we uncovered in this work was successfully applied to enhance E. coli's furfural tolerance by heterologous expression of ctADH1. The study provides deeper insights into strain modification for biofuel production by efficient lignocellulose fermentation.
\end{abstract}

Keywords: Candida tropicalis, Furfural tolerance, Furfural detoxification, Alcohol dehydrogenase 1

\footnotetext{
*Correspondence: yuanqp@mail.buct.edu.cn

${ }^{1}$ State Key Laboratory of Chemical Resource Engineering, College of Life Science and Technology, Beijing University of Chemical Technology, West Room 314, Science and Technology Building, No. 15 North Third Ring East Road, Chaoyang District, Beijing 100029, People's Republic of China

Full list of author information is available at the end of the article
} 


\section{Background}

Lignocellulosic biomass is an attractive and promising substrate source for the production of biofuels and value-added chemicals. Pretreatment is a primary and key step for efficient and economic bioconversion of lignocellulosic biomass [1]. However, during pretreatment of lignocellulose by acid hydrolysis, steam explosion, and high-temperature steaming [2-5], several groups of inhibitors for fermentation are generated, such as furfural, phenolic compounds, and acetic acid [6-8]. Furfural, derived from the dehydration of pentose, is one of the primary inhibitors in hemicellulose hydrolysate. Furfural negatively affected specific growth rate of cells [9] and acted as inhibitor to glycolytic enzymes [7]. Moreover, furfural could increase the toxicity of acetate in yeasts [10] and the toxicity of phenols in Escherichia coli $[11,12]$. In Saccharomyces cerevisiae, furfural has been shown to cause an accumulation of reactive oxygen species and cellular damage to mitochondria, vacuoles, actin, and nuclear chromatin [13]. In Candida tropicalis, growth and xylitol fermentation could also be inhibited by furfural [14].

To reduce the inhibition caused by furfural, recent research has been focused on the mechanisms of tolerance and detoxification in microbes. However, resistance phenotypes usually involve complicated multi-genic regulations among stress responses [15]. Silencing of oxidoreductase genes increased furfural tolerance in $E$. coli LY180 [16]. Overexpression of propanediol oxidoreductase and transhydrogenase improved the growth of $E$. coli in the presence of furfural $[17,18]$. Recent research showed that overexpression of alcohol dehydrogenases (encoded by $A D H$ ), transcription activator Msn2, oxidative stress regulator Yap1, and glucose-6-phosphate dehydrogenase, which were confirmed by transcriptional analysis, proteomic analysis, and disruption library screening, could improve furfural tolerance in yeast [19-22]. In S. cerevisiae, furfural could be converted into furfuryl alcohol by NADH-dependent alcohol dehydrogenase 1 [23-25]. Co-expression of transaldolase 1 and alcohol dehydrogenase 1 in recombinant xylose-fermenting $S$. cerevisiae improves the production of ethanol and xylitol in xylose medium in the presence of furfural [26]. However, furfuryl alcohol is not stable, and the detection of this compound is complicated for its polymerization [27] and degradation [28].

In our previous study, C. tropicalis was used to produce xylitol from hemicellulose hydrolysate [2]. The metabolic responses caused by complex inhibitors (including furfural, acetic acid, and phenol) in C. tropicalis have been studied by a gas chromatography/mass spectrum-based metabolomics approach [29]. We also found that C. tropicalis showed faster furfural detoxification rate in xylose medium than in glucose medium [30]. However, C. tropicalis's resistance to furfural has not been evaluated. The mechanism of furfural tolerance and metabolic responses to furfural were still unknown for $C$. tropicalis. In this study, we found that $C$. tropicalis showed a comparable intrinsic tolerance to furfural by half maximal inhibitory concentration $\left(\mathrm{IC}_{50}\right)$ analysis. To provide deeper insights into the mechanisms of furfural tolerance and metabolic responses, we investigated the expression of key genes involved in ethanol production using quantitative real-time PCR (qRT-PCR). We found that $A D H 1$ from C. tropicalis (ctADH1) played a key role in the resistance to furfural, and furfural triggered a series of complex responses in C. tropicalis to eliminate furfural and maintain redox balance. Furthermore, E. coli's furfural tolerance was successfully enhanced by heterologous expression of $c t A D H 1$. The mechanism of $C$. tropicalis's furfural tolerance elucidated in this work will provide useful information for metabolic engineering of efficient strains for lignocellulose fermentation.

\section{Methods}

Strains, medium, and plasmids

Wild-type C. tropicalis (Strain No. 2.1776, China General Microbiological Culture Collection Center, China) was employed in fermentation. In our previous study, we obtained uracil auxotroph C. tropicalis YE (ura3/ura3) derived from wild-type $C$. tropicalis by chemical mutagenesis. C. tropicalis $\mathrm{YE}$ was used as the host strain for gene disruption. E. coli DH5 $\alpha$ was used as the host strain for plasmid construction and propagation. $E$. coli BL21 (DE3) was used for protein expression. When needed, ampicillin and kanamycin were added into the medium with the final concentration of $100 \mu \mathrm{g} / \mathrm{mL}$. The details of strains used in this study are depicted in Additional file 1: Table S1.

Preculture medium was used for inoculation. Xylose medium was used for toxicity test, tolerance test, $\mathrm{IC}_{50}$ analysis, transcriptional analysis, and HPLC analysis of C. tropicalis. The fermentation was carried out at $30{ }^{\circ} \mathrm{C}$ and $200 \mathrm{rpm}$ in $250-\mathrm{ml}$ flask for C. tropicalis. Yeast extract peptone dextrose (YPD) medium, yeast nitrogen base without amino acids (YNB) medium, YNB-uracil (YNB-URA) medium, and YNB-URA-5-fluoroorotic acid (YNB-URA-5FOA) medium were used for genetic manipulation of $C$. tropicalis. Luria-Bertani (LB) medium was used for inoculants, propagation, and genetic manipulation of E. coli. Modified minimal basal salts (M9) medium was used for furfural degradation experiment of $E$. coli. The fermentation was carried out at $37^{\circ} \mathrm{C}$ and $200 \mathrm{rpm}$ in $250-\mathrm{ml}$ flask for E. coli. pCS27 and pETDuet-1 are high- and medium-copy plasmids, respectively. They were both used for protein expression. 
The details of plasmids used in this study are depicted in Additional file 1: Table S1. The constituents of medium were described in Additional material.

\section{Growth curves, $\mathrm{IC}_{50}$, and cell viability test under furfural stress}

In furfural tolerance tests, furfural of $0,1,3,5,7$, and $9 \mathrm{~g} / \mathrm{L}$ was added at the beginning of fermentation. The optical density at $600 \mathrm{~nm}\left(\mathrm{OD}_{600}\right)$ of the biomass was measured by UV spectrophotometer (Shimadzu UV-2450, China) at $600 \mathrm{~nm}$ after dilution. The growth curves of $C$. tropicalis were determined by $\mathrm{OD}_{600}$. The values of $\mathrm{IC}_{50}$ were calculated by Graphpad Prism probit analysis (GraphPad Software, USA).

Methylene blue staining was used to evaluate C. tropicalis's cell viability in the presence of furfural. In cell viability analysis, furfural of $1,3,5,7$, and $9 \mathrm{~g} / \mathrm{L}$ was added into the culture at mid-exponential phase $(6 \mathrm{~h}$ after inoculation), when the cells showed the most significant metabolic responses to stress. Cells were harvested and dropped onto a clean glass slide. Methylene blue solvent $(0.1 \%)$ was added to the cells. After having been stained for $30 \mathrm{~min}$, cells were observed by optical microscopy (Olympus CX 23, Japan). The dead cells were stained dark blue, while the living cells were transparent.

\section{Fermentation liquor analysis and redox balance analysis}

In fermentation experiments, furfural of 3,5 , and $7 \mathrm{~g} / \mathrm{L}$ was added to the fermentation at mid-exponential phase (6 $\mathrm{h}$ after inoculation) in furfural-treated groups. After centrifugation, samples were filtered through a $0.22-\mu \mathrm{m}$ filter. The supernatant was diluted before being analyzed by high-performance liquid chromatography (HPLC). The analysis of xylose, xylitol, and furfural were performed with a Hitachi HPLC system (Hitachi Chromaster, Japan). They were separated by an Aminex HPX-87H column (Bio-Rad, USA) at $45^{\circ} \mathrm{C}$ and detected by a refractive index detector. $5 \mathrm{mM} \mathrm{H} \mathrm{H}_{4}$ was used as mobile phase at a flow rate of $0.6 \mathrm{~mL} / \mathrm{min}$. The ratio of NADH/ $\mathrm{NAD}^{+}$in the samples were extracted and measured by $\mathrm{NADH} / \mathrm{NAD}^{+}$Assay Kit (BioAssay Systems, USA).

\section{RNA extraction, CDNA synthesis, and qRT-PCR}

TRIzol Reagent (Invitrogen, USA) was used to extract total RNA from cells pellets by liquid nitrogen grinding. RNA samples were quantified by SmartSpec plus (Biorad). RNA quality was determined by electrophoresis. We employed reverse transcription with random hexamer primed reactions by M-MLV First Strand cDNA Synthesis Kit (Omega Bio-Tek, China). The cDNA was stored at $-20{ }^{\circ} \mathrm{C}$. Relative quantification of cDNA was performed by Perfectstart SYBR Green qPCR master mix (Omega Bio-Tek) on a Mastercycler Realplex2 cycler
(Eppendorf, Germany). The primers used in qRT-PCR are listed in Additional file 1: Table S2. Samples were analyzed in triplicates, with negative controls in each assay. The expression of related genes was calculated relative to the expression of ACTIN by the comparative $\mathrm{C}_{\mathrm{T}}$ method. $T$ tests were performed by SPSS 19.0 (SPSS Inc., USA).

\section{Construction of $c t A D H 1$ disruption cassettes, transformation, and sensitivity experiment}

Gene disruption of $C$. tropicalis was performed by homologous recombination [31, 32]. The URA3 gene (with promoter and terminator, GenBank accession number AB006207.1) was amplified by PCR from the genomic DNA of wild-type C. tropicalis with primers URA3-F and URA3-R [31]. The $c t A D H 1$ gene (NCBI Reference Sequence: XM_002546589.1) and ctADH1m (the middle part of $c t A D H 1,0.55 \mathrm{~kb}$ ) were amplified by PCR from the genomic DNA of uracil auxotroph C. tropicalis YE with primers ADH1-F-ADH1-R and ADH1mF-ADH1m-R, respectively. hisG1 fragments $(1.1 \mathrm{~kb})$ and his 62 fragments $(1.1 \mathrm{~kb})$ were amplified by PCR from the plasmid pCUB6 [33] with two sets of primers, hisG1-FhisG1-R and hisG2-F-hisG2-R, respectively. $c t A D H 1$ and ctADH1m were inserted into T-Vector pMD19 (Simple), and the plasmid was designated Ts-ADH1 and TsADH1m, respectively.

ADH1-Ts-ADH1 was amplified by PCR from TsADH1 with Ts-Ar-F and Ts-Ar-R. URA3 digested by SacI and PstI was ligated with ADH1-Ts-ADH1 digested by $S a c \mathrm{I}$ and PstI. The resulting plasmid was designated Ts-ADH1-URA3. hisG1 and hisG2 were inserted into Ts-ADH1-URA3 one by one, resulting in plasmid TsAUH and Ts-AUHH. ADH1-hisG1-URA3-hisG2-ADH1, which was used as the first disruption cassette, was amplified from Ts-AUHH with ADH1-F and ADH1-R. ADH1m-Ts-ADH1m was amplified by PCR from TsADH1m with Ts-Amr-F and Ts-Amr-R. URA3 digested by $S a c \mathrm{I}$ and PstI, was ligated with ADH1m-Ts-ADH1m digested by $S a c I$ and PstI. The resulting plasmid was designated Ts-AmU. ADH1m-URA3-ADH1m, which was used as the second disruption cassette, was amplified from Ts-ADH1m-URA3 with ADH1m-F and ADH1m-R.

Candida tropicalis was transformed using the $\mathrm{LiCl}$ method [34]. The first disruption cassette ADH1-hisG1URA3-hisG2-ADH1 was transformed into C. tropicalis YE. The transformants (C. tropicalis T1) selected on YNB plates were confirmed by PCR. The transformants with URA3 marker were spread on YNB-URA-5FOA plates. The URA3 pop-out mutants (C. tropicalis $\mathrm{T} 2$ and C. tropicalis T3) were selected from the 5-FOA-resistant colonies using PCR. The second disruption cassette was transformed into C. tropicalis $\mathrm{T} 2$, and the resulting cells were selected by YNB plate. The transformants $(C$. 
tropicalis T4) were confirmed by PCR. The primers used in this study are listed in Additional file 1: Table S2.

To verify the furfural sensitivity of $C$. tropicalis, cells of C. tropicalis T4, T3, T2, and YE were inoculated into $15-\mathrm{ml}$ glass tubes containing $5 \mathrm{ml}$ of YPD and $3 \mathrm{~g} / \mathrm{L}$ furfural. The tubes were incubated for $10 \mathrm{~h}$ at $30^{\circ} \mathrm{C}$ and $200 \mathrm{rpm}$.

\section{Heterologous expression of $c t A D H 1$ and in vivo furfural degradation in E. coli}

ctADH1 was subcloned into pETDuet-1 by SacI and $K p n \mathrm{I}$, and into pCS-27 by SalI and SmaI, resulting plasmid pET-ADH1 and pCS-ADH1. The primers used in this study are listed in Additional file 1: Table S2. To evaluate alcohol dehydrogenase 1's activity of degrading furfural, the plasmid pET-ADH1, pETDuet-1, pCS$\mathrm{ADH} 1$, and pCS-27 were transformed into E. coli BL21 (DE3). The resultant transformants (E. coli PE, E. coli PC, E. coli PEA, and E. coli PCA) were inoculated in $5 \mathrm{~mL}$ M9 medium and cultured at $37{ }^{\circ} \mathrm{C}$. The overnight cultures were inoculated into $50 \mathrm{~mL} \mathrm{M9} \mathrm{medium.} \mathrm{The}$ medium was cultured at $37{ }^{\circ} \mathrm{C}$. When $\mathrm{OD}_{600}$ reaching 0.6 , the cultures were induced by $0.25 \mathrm{mM}$ of $\beta-\mathrm{D}-1$ thiogalactopyranoside (IPTG) at $30{ }^{\circ} \mathrm{C}$. Furfural of $1 \mathrm{~g} / \mathrm{L}$ was added into the culture $6 \mathrm{~h}$ after inoculation. The concentration of furfural was measured by HPLC. The expression of $c t A D H 1$ was confirmed by SDS-PAGE analysis [35].

\section{Results and discussion}

\section{Tolerance and in situ detoxification of $C$. tropicalis under furfural stress}

In this study, furfural tolerance and cell viability of furfural in C. tropicalis were evaluated by growth curves, $\mathrm{IC}_{50}$, and methylene blue staining. To determine the growth inhibition caused by furfural, furfural of $0,1,3,5$, 7 , and $9 \mathrm{~g} / \mathrm{L}$ was added into the medium at the beginning of fermentation. The lag phase of $C$. tropicalis in growth curves was significantly prolonged by furfural (Fig. 1). Interestingly, even though $1 \mathrm{~g} / \mathrm{L}$ furfural prolonged the lag phase of $C$. tropicalis, the final biomass produced in the medium containing $1 \mathrm{~g} / \mathrm{L}$ furfural was slightly greater than that in the control (Fig. 1). It was reported that furfural of $10 \mathrm{mM}$ (approximately $1 \mathrm{~g} / \mathrm{L}$ ) slightly increased Thermoanaerobacter pseudethanolicus's cell yield by $11 \%$ [35]. Furfural could replace glycerol as an electron sink and allow less carbon loss to byproducts and more carbon distribution towards biomass synthesis [36]. However, $C$. tropicalis could hardly grow up in the presence of $5 \mathrm{~g} / \mathrm{L}$ furfural at the beginning of fermentation (Fig. 1). C. tropicalis's $\mathrm{IC}_{50}$ (Table 1) for furfural was calculated by growth curves (Fig. 1). C. tropicalis's $\mathrm{IC}_{50}$ for furfural was $3.69 \mathrm{~g} / \mathrm{L}$ at $24 \mathrm{~h}$. To our knowledge, the $\mathrm{IC}_{50}$ of

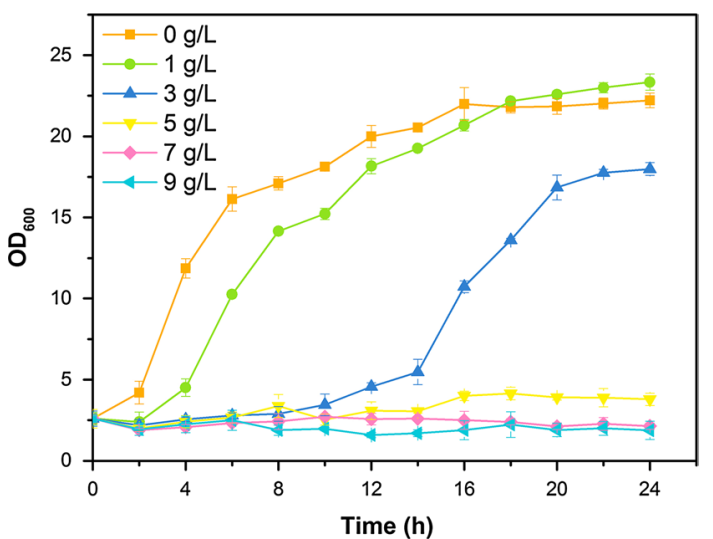

Fig. 1 Growth profiles during fermentation in the presence of furfural. Furfural of $0,1,3,5,7$, and $9 \mathrm{~g} / \mathrm{L}$ was added into xylose medium at the beginning of fermentation. The error bars represent the standard deviation calculated from triplicates

C. tropicalis was higher than most wild-type microbes reported in the literature (Table 1 ). Although the direct comparisons of $\mathrm{IC}_{50}$ between different organisms are difficult due to the differences in the culture conditions, our results suggest that $C$. tropicalis possesses a comparable tolerance to furfural.

Methylene blue staining was used to evaluate cell viability. Furfural of $1,3,5,7$, and $9 \mathrm{~g} / \mathrm{L}$ was added at midexponential phase ( $6 \mathrm{~h}$ after inoculation). Cell viability test (Table 2; Additional file 1: Figure S1) by methylene blue staining showed that quite few cells died at an early stage of furfural treatment $(0.5 \mathrm{~h}$ after addition of furfural). With the increase of treatment time, the proportion of dead cells increased until depletion of furfural. Furfural caused more cell death $1 \mathrm{~h}$ and $2.5 \mathrm{~h}$ after furfural treatment. However, the proportion of living cells increased $5 \mathrm{~h}$ after furfural treatment. In the presence of $1,3,5$, and $7 \mathrm{~g} / \mathrm{L}$ furfural, $C$. tropicalis could still reproduce, and living cells were dominant as shown by the cell viability test (Table 2; Additional file 1: Figure S1), showing comparable robustness to furfural. However, $9 \mathrm{~g} / \mathrm{L}$ furfural caused more than 50 and $90 \%$ cells dead 1 and $2.5 \mathrm{~h}$ after furfural treatment (Table 2; Additional file 1: Figure S1), respectively. The result indicated that $9 \mathrm{~g} / \mathrm{L}$ furfural was lethal for C. tropicalis. The result of methylene blue staining was also used to determine the appropriate concentration of furfural used in the following experiments, since furfural of high concentrations would cause cell death directly [19]. After cell death, the analysis of fermentation liquor would provide poor information, and RNA in cells would degrade soon. Therefore, it was determined that furfural of 3,5 , and $7 \mathrm{~g} / \mathrm{L}$ was suitable for analysis of the transcription and fermentation liquor. 
Table $1 \mathrm{IC}_{50}(\mathrm{~g} / \mathrm{L})$ for furfural in different microorganisms

\begin{tabular}{|c|c|c|c|c|c|}
\hline Organism & $I C_{50}$ for furfural & Carbon source (w/v) & Time (h) & Temp $\left({ }^{\circ} \mathrm{C}\right)$ & Ref. \\
\hline C. tropicalis YE & 3.69 & $10 \%$ xylose & 24 & 30 & This study \\
\hline C. shehatae & 1.02 & $2 \%$ xylose & 32 & 26 & {$[36]$} \\
\hline Pichia stipitis & 0.85 & $2 \%$ xylose & 32 & 26 & {$[36]$} \\
\hline Zymomonas. mobilis & 1.93 & $2 \%$ glucose & 24 & 30 & {$[36]$} \\
\hline S. cerevisiae CBS 1200 & 0.51 & $2 \%$ glucose & 24 & 26 & {$[36]$} \\
\hline S. cerevisiae NSI 113 & 2 & $1 \%$ glucose & 48 & 30 & {$[37]$} \\
\hline Mixed culture from anaerobic sludge & 1.05 & $4 \%$ glucose & 48 & 37 & {$[38]$} \\
\hline Bacillus coagulans & $2.5-5$ & $5-10 \%$ glucose & 24 & 50 & [39] \\
\hline Caldicellulosiruptor saccharolyticus & $1-2$ & $1 \%$ glucose & 16,40 & 72 & {$[40]$} \\
\hline Thermotoga neapolitana & $2-4$ & $1 \%$ glucose & 16,40 & 80 & {$[40]$} \\
\hline E. coli & $2.4,2.9$ & $5 \%$ xylose & 24,48 & 37 & {$[11]$} \\
\hline Thermoanaerobacter pseudethanolicus & 3 & $0.7 \%$ glucose & 12 & 65 & {$[41]$} \\
\hline Thermoanaerobacter pseudethanolicus & $3-4$ & $0.7 \%$ glucose & 24 & 65 & {$[41]$} \\
\hline
\end{tabular}

Table 2 Ratio of dead cells to total cells counted by the result of methylene blue staining

\begin{tabular}{lccccc}
\hline Time after furfural treatment & \multicolumn{4}{l}{ Ratio of dead cells to total cells } \\
\cline { 2 - 6 } & $\mathbf{1} \mathbf{g} / \mathbf{L}$ furfural & $\mathbf{3 ~ g / L ~ f u r f u r a l}$ & $\mathbf{5 ~ g / L ~ f u r f u r a l}$ & $\mathbf{7 ~ g / L ~ f u r f u r a l}$ & $\mathbf{9} \mathbf{~ g / L ~ f u r f u r a l}$ \\
\hline $30 \mathrm{~min}$ & $1.6 \%(2 / 126)$ & $3.3 \%(5 / 152)$ & $2.3 \%(5 / 218)$ & $4.2 \%(9 / 213)$ & $11.7 \%(24 / 206)$ \\
$1 \mathrm{~h}$ & $1.5 \%(2 / 129)$ & $3.3 \%(5 / 151)$ & $7 \%(10 / 142)$ & $25 \%(45 / 180)$ & $61.5 \%(40 / 65)$ \\
$2.5 \mathrm{~h}$ & $2.3 \%(2 / 870)$ & $5.6 \%(10 / 178)$ & $18.2 \%(18 / 99)$ & $25.8 \%(63 / 244)$ & $95.1 \%(136 / 143)$ \\
$5 \mathrm{~h}$ & $1 \%(1 / 104)$ & $1 \%(1 / 101)$ & $14.9 \%(23 / 154)$ & $21 \%(35 / 167)$ & $93.3 \%(70 / 75)$ \\
\hline
\end{tabular}

The results of growth curves, $\mathrm{IC}_{50}$, and cell viability test indicated that $C$. tropicalis exhibited comparable tolerance under furfural stress. The proportion of living cells increased $5 \mathrm{~h}$ after furfural treatment, suggesting that the stress caused by furfural was reduced $5 \mathrm{~h}$ after furfural treatment. The reduced furfural stress was probably due to the degradation and detoxification of furfural.

In fermentation liquor analysis, when adding furfural of 3,5 , and $7 \mathrm{~g} / \mathrm{L}$ into the culture at mid-exponential phase ( $6 \mathrm{~h}$ after inoculation), the rate of furfural degradation reached $3,3.33$, and $2.80 \mathrm{gL}^{-1} \mathrm{~h}^{-1}$, respectively. Furfural degradation rate was calculated by the following equation:

$$
\text { Furfural degradation rate }\left(\mathrm{gL}^{-1} \mathrm{~h}^{-1}\right)=\frac{C_{f}}{T_{2}-T_{1}},
$$

where $C_{f}, T_{1}$, and $T_{2}$ refer to initial furfural concentration, time of furfural addition, and time of furfural depletion, respectively.

Furfural was all depleted in $3 \mathrm{~h}$ after addition of furfural at mid-exponential phase (Fig. 2). Degradation rate of furfural in the groups treated by $5 \mathrm{~g} / \mathrm{L}$ furfural was even faster than that treated by $3 \mathrm{~g} / \mathrm{L}$ furfural. It suggested that furfural of medium concentration $(5 \mathrm{~g} / \mathrm{L})$ might induce faster and stronger metabolic responses,

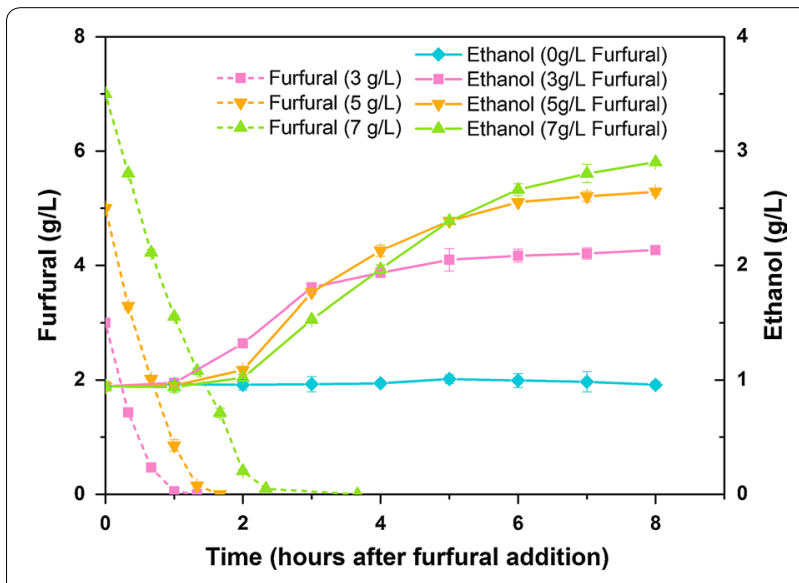

Fig. 2 Furfural and ethanol profiles during fermentation in the presence of furfural. Furfural of $0,3,5$, and $7 \mathrm{~g} / \mathrm{L}$ was added into xylose medium at mid-exponential phase. The error bars represent the standard deviation calculated from triplicate experiments

and result in faster degradation rate, than furfural of low concentration (3 g/L). However, furfural of high concentration $(7 \mathrm{~g} / \mathrm{L})$ was too toxic to induce efficient metabolic responses. As shown in Fig. 2, the degradation of furfural was followed by ethanol production. As soon as the 
furfural was depleted, ethanol concentration started to increase. In addition, the concentration of ethanol produced in the fermentation increased with the concentration of furfural added in the medium. It was proposed that the depletion of furfural triggered ethanol production. The levels of ethanol in furfural-treated groups showed more than twofold increase compared to those in control groups $(1 \mathrm{~g} / \mathrm{L})$. The production of ethanol was probably relevant to the time of furfural degradation and the concentration of furfural added in the medium.

From above results, we presumed that the enzymes which were induced by furfural could also catalyze the production of ethanol. In other words, the enzymes involved in ethanol biosynthesis might play a key role in metabolic responses to furfural or furfural detoxification. In $C$. tropicalis, the enzyme directly involved in ethanol biosynthesis was alcohol dehydrogenase 1 (encoded by ctADH1). To validate the hypothesis, qRT-PCR, redox balance analysis, gene knock-out experiment, and overexpression of alcohol dehydrogenase 1 were carried out in this study.

\section{Metabolic responses of $C$. tropicalis under furfural stress}

In C. tropicalis, xylose is firstly reduced into xylitol by xylose reductase which uses both NADPH and NADH as cofactors [42]. Subsequently xylitol dehydrogenase almost exclusively uses $\mathrm{NAD}^{+}$as cofactor in the oxidation of xylitol into xylulose [43]. After phosphorylation, xylulose is utilized by the pentose phosphate pathway to provide a carbon source and energy for cells. Furfural of various concentrations showed different effects on the consumption of xylose, when furfural was added $6 \mathrm{~h}$ after inoculation. Furfural of lower concentration ( 3 and $5 \mathrm{~g} / \mathrm{L}$ ) did not significantly inhibit the consumption rate of xylose (Fig. 3), indicating that the rate of xylose transportation or xylose metabolism was not affected by furfural of low concentrations. Low concentrations of furfural even slightly promoted xylose consumption rate at $28 \mathrm{~h}$. After addition of furfural, xylitol accumulation was considerably decreased and delayed (Fig. 3). The more furfural was added, the less xylitol was accumulated. Xylitol concentration in the groups treated by $7 \mathrm{~g} / \mathrm{L}$ furfural was decreased by $15 \%$ compared to the control. These results indicated that the proportion of xylitol consumed for providing energy and intermediate metabolites increased, and less xylitol was accumulated after furfural treatment. Hyung-Min et al. reported that the E. coli strain with greater furfural detoxification rate also showed a greater sugar consumption rate [44]. Ask et al. found that furfural stress could induce energy-requiring repair mechanism, and the energy charge was slightly higher after addition of furfural compared to the control [45]. Our previous study also showed that complex inhibitors could

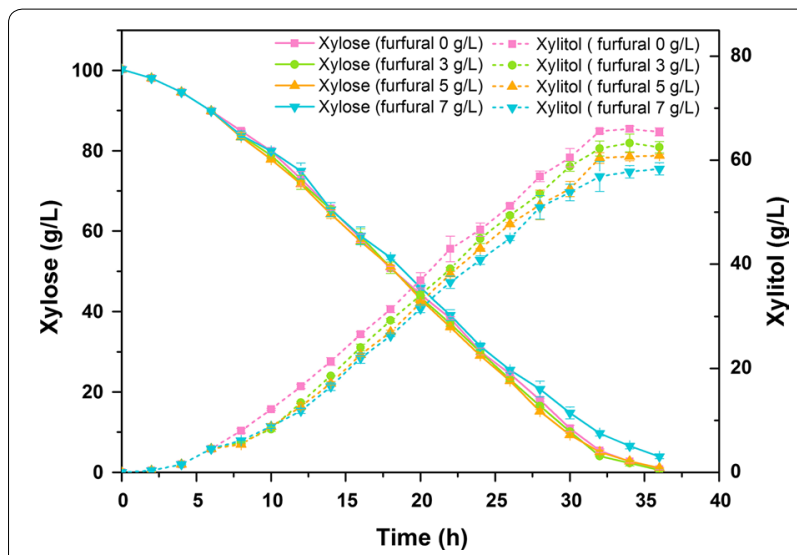

Fig. 3 Xylose and xylitol profiles during fermentation in the presence of furfural. Furfural of $0,3,5$, and $7 \mathrm{~g} / \mathrm{L}$ was added into xylose medium at mid-exponential phase. The error bars represent the standard deviation calculated from triplicate experiments

enhance TCA cycle to provide more energy and metabolic intermediates for inhibitor tolerance [29]. However, when adding furfural of high concentration $(7 \mathrm{~g} / \mathrm{L})$ into the medium, xylose consumption rate was severely inhibited (Fig. 3), indicating that the xylose transportation or xylose metabolism was blocked by furfural of high concentrations. The flux of the pentose phosphate pathway would decrease accordingly.

In the groups treated by furfural of 3, 5, and $7 \mathrm{~g} / \mathrm{L}$, the transcription of gene $c t A D H 1$ showed the same transcriptional pattern (Fig. 4). The transcription of $c t A D H 1$ was increased by furfural treatment. After furfural was totally degraded, $c t A D H 1$ showed a decreased transcriptional level. This indicated that the expression of $c t A D H 1$ in C. tropicalis was remarkably induced by furfural as metabolic responses, and after the depletion of furfural the transcription of $c t A D H 1$ was immediately restrained (Fig. 4). Thus, we presumed that alcohol dehydrogenase 1 (encoded by $c t A D H 1$ ) was relevant to furfural degradation.

In the present study, the NADH/NAD ${ }^{+}$ratio was used to determine the change of redox balance. NADH/NAD ${ }^{+}$ ratio in $C$. tropicalis decreased after furfural treatment compared with the control (Fig. 5). Even after the depletion of furfural, $\mathrm{NADH} / \mathrm{NAD}^{+}$ratio in $C$. tropicalis with furfural was still lower than those in the control, suggesting that the process of furfural degradation probably consumed the coenzyme NADH, and the disturbance of redox balance caused by furfural was long-lasting.

It was reported that the $\mathrm{NADH} / \mathrm{NAD}^{+}$ratio was decreased by furfural degradation in $S$. cerevisiae as a result of NADH consumption [19]. The results provided more evidence to prove that furfural was probably reduced by alcohol dehydrogenase 1 with NADH as 


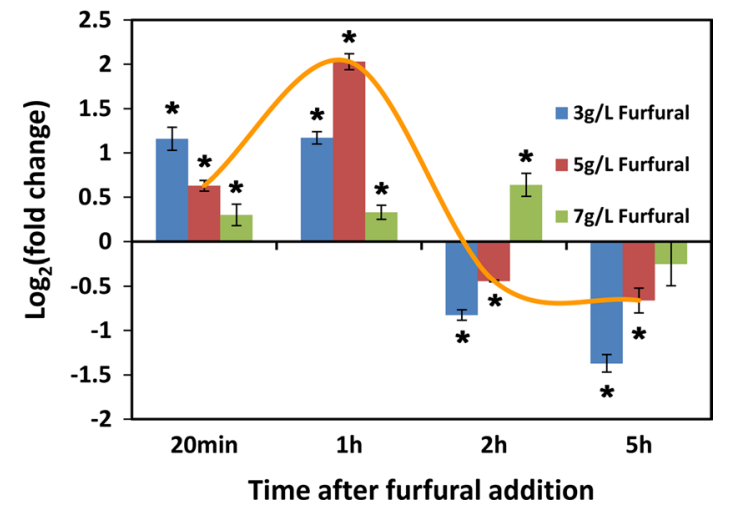

Fig. 4 Transcription analysis of $C t A D H 1$ by qRT-PCR after furfural treatment. Furfural of $0,3,5$, and $7 \mathrm{~g} / \mathrm{L}$ was added into xylose medium at mid-exponential phase. The error bars represent the standard deviation calculated from triplicate experiment. ${ }^{*} p<0.05$ compared with the control

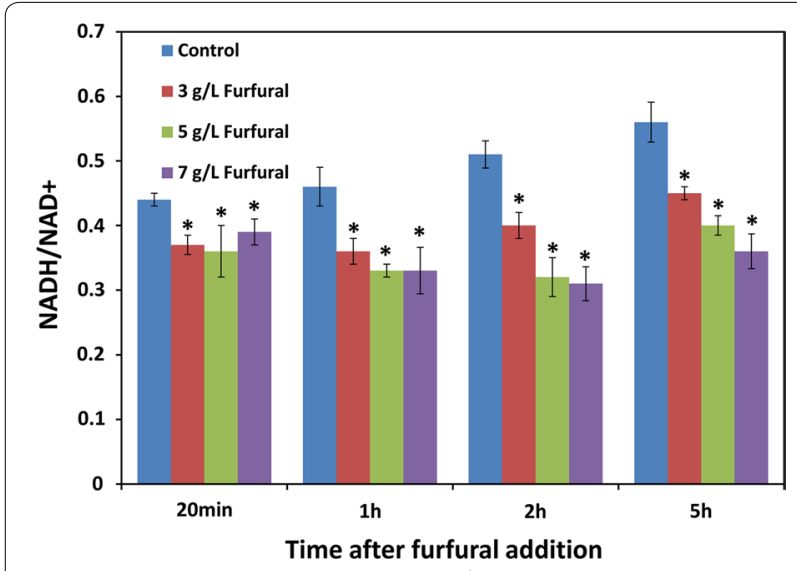

Fig. 5 Ratio of intracellular NADH/NAD ${ }^{+}$in the presence of furfural. Furfural of $0,3,5$, and $7 \mathrm{~g} / \mathrm{L}$ was added into xylose medium at midexponential phase. The error bars represent the standard deviation calculated from triplicate experiments. ${ }^{*} p<0.05$ compared with the control

coenzyme in C. tropicalis. It was also reported that the reduction of furfural to furfuryl alcohol might be catalyzed by alcohol dehydrogenases with NADH as a cofactor in S. cerevisiae, and glycolysis was possibly activated by furfural to provide more $\mathrm{NADH}$ as is required for the reduction of furfural $[19,46]$. Furfuryl alcohol was unstable and less toxic than furfural.

\section{Role of $c t A D H 1$ played in furfural detoxification}

To confirm the function of $c t A D H 1$, we used homologous recombination system to disrupt the gene $c t A D H 1$ [31]. Since $C$. tropicalis was diploid, cassettes were integrated into the two copies of $c t A D H 1$ fragment region in the $C$. tropicalis genome one by one. The site-specific insertion of the transformants was confirmed by PCR (Additional file 1: Figure S2).

To verify the function of $A D H 1$ in $C$. tropicalis, cells of $C$. tropicalis T4, T3, T2, and YE were inoculated into YPD medium containing $3 \mathrm{~g} / \mathrm{L}$ furfural. C. tropicalis $\mathrm{T} 4$ could hardly grow up in YPD medium containing $3 \mathrm{~g} / \mathrm{L}$ furfural after the knockout of $c t A D H 1$ (Additional file 1: Figure S3). Furfural tolerance of $C$. tropicalis T3 and $C$. tropicalis $\mathrm{T} 2$ was also weakened after the destruction of ctADH1 in the first chromosome (Additional file 1: Figure S3). However, the parent strain C. tropicalis YE showed better growth in the presence of furfural compared with $c t A D H 1$ disruption strains. This result proved that C. tropicalis's robustness and tolerance to furfural were related to alcohol dehydrogenase 1 . Laadan et al. found that HMF and furfural could be reduced by $\mathrm{NADH}$ dependent alcohol dehydrogenase 1 in S. cerevisiae [25].

To further confirm the function of $c t A D H 1$, alcohol dehydrogenase 1 was overexpressed in E. coli. ctADH1 was inserted into the vector pETDuet- 1 and pCS-27. The insertion was confirmed by DNA sequencing of the PCR product. The expression of alcohol dehydrogenase 1 in $E$. coli PEA and E. coli PCA was confirmed by SDS-PAGE analysis (Additional file 1: Figure S4).

The rate of furfural degradation and detoxification was an indication of tolerance to furfural. When cultured in M9 medium, E. coli PEA and E. coli PCA showed much stronger ability of furfural degradation (Additional file 1: Figure S5A, B) than the control. The rate of furfural degradation in E. coli BL21 (DE3) with pET-ADH1 and pCS-ADH1 was increased by 1.59 -fold and 1.28 -fold compared to the control, respectively, indicating that overexpression of $c t A D H 1$ improved $E$. coli's ability of detoxicating furfural. The ability of degrading furfural in $E$. coli increased with the level of $c t A D H 1$ expression. This result confirmed that alcohol dehydrogenase 1 from $C$. tropicalis could degrade furfural. Hasunuma also found that overexpression of NADH-dependent alcohol dehydrogenase 1 could improve ethanol fermentation in the presence of furfural in S. cerevisiae [24]. Moreover, heterologous expression of $c t A D H 1$ in $E$. coli resulted in stronger furfural detoxification, which was a promising application of $c t A D H 1$ for the production of lignocellulosic biofuel.

\section{The mechanism of furfural detoxification in C. tropicalis}

To determine the mechanism of induction of alcohol dehydrogenase 1 by furfural, the effect of acetaldehyde on transcription of $c t A D H 1$ was analyzed by qRT-PCR. Interestingly, the transcriptional response of $c t A D H 1$ in $C$. tropicalis treated by acetaldehyde $(2 \mathrm{~g} / \mathrm{L})$ showed similar pattern to that in $C$. tropicalis treated by furfural (Figs. 4, 6a). Alcohol dehydrogenase 1 is known for 

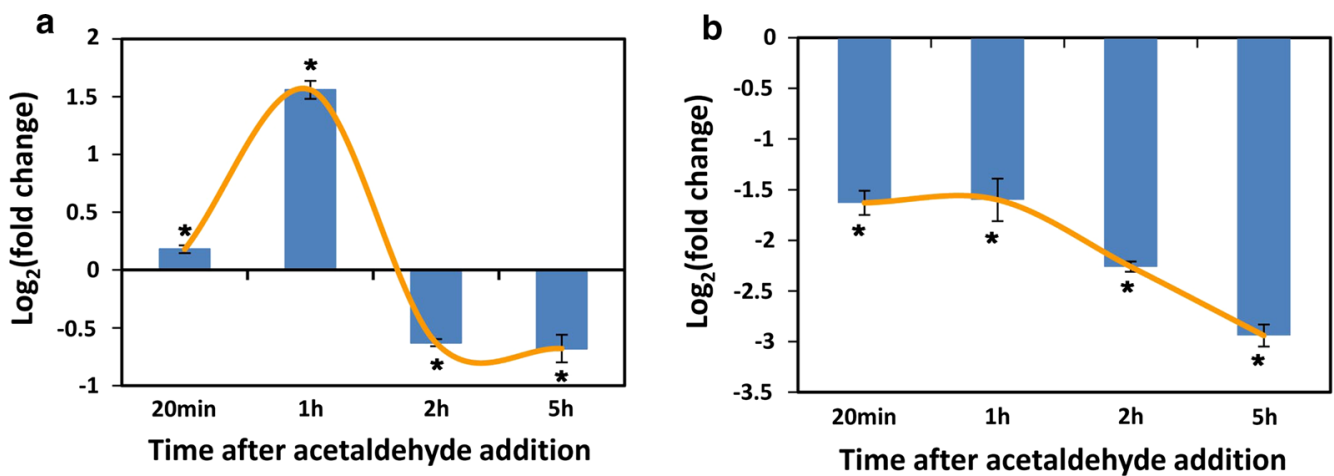

Fig. 6 Acetaldehyde and ethanol regulated the transcription of $c t A D H 1$. a Transcription analysis of ctADH1 after acetaldehyde addition; b Transcription analysis of $c t A D H 1$ after ethanol addition. Acetaldehyde or ethanol of $2 \mathrm{~g} / \mathrm{L}$ was added into the xylose medium culture at mid-exponential phase. The error bars represent the standard deviation calculated from triplicate experiments. ${ }^{*} p<0.05$ compared with the control

catalyzing the transformation from acetaldehyde to ethanol [47]. The gene $c t A D H 1$ was up-regulated after acetaldehyde addition. However, the production of ethanol would consume reducing power. After the depletion of extra acetaldehyde, the transcriptional level of $c t A D H 1$ decreased to restrain ethanol production and maintain redox balance. Furfural has the same functional groupaldehyde as acetaldehyde, which might lead to the same signal transduction and the same active site in alcohol dehydrogenase 1. Furfural and acetaldehyde caused the same transcriptional responses of $c t A D H 1$ in C. tropicalis, which further supported the hypothesis that furfural was degraded by alcohol dehydrogenase 1 in C. tropicalis. However, after depletion of furfural, alcohol dehydrogenase 1 still remained in the cell. Subsequently, ethanol was produced by alcohol dehydrogenase 1 , leading to the increase of ethanol level in the medium (Fig. 2). As enzyme, alcohol dehydrogenase 1 was not stable in the cell. After the degradation of alcohol dehydrogenase 1, the production of ethanol reached stationary phase. To determine the effect of ethanol on the transcription of $c t A D H 1$, ethanol of $2 \mathrm{~g} / \mathrm{L}$ was added into the medium $6 \mathrm{~h}$ after inoculation. qRT-PCR analysis showed that the transcription of $c t A D H 1$ was down-regulated $20 \mathrm{~min}$, 1 , and $2 \mathrm{~h}$ after addition of ethanol (Fig. $6 \mathrm{~b}$ ). The downregulation of $c t A D H 1$ after furfural depletion could help cells to avoid the loss of energy and carbon source, since alcohol dehydrogenase 1 is no more required for furfural detoxification. The repression of $c t A D H 1$ could also minimize ethanol production and NADH consumption, making up for the imbalance of $\mathrm{NADH} / \mathrm{NAD}^{+}$ratio caused by furfural.

The transcription of $c t A D H 1$ was regulated by both furfural and ethanol (Fig. 7). The induction of $c t A D H 1$ was triggered by furfural to decrease toxic stress, which was followed by ethanol production. After furfural depletion, the transcription of $c t A D H 1$ was repressed by ethanol to produce less ethanol and maintain redox balance. The regulation system of $c t A D H 1$ minimized the toxic stress of furfural and oxidative stress caused by furfural detoxification and ethanol production.

\section{Conclusions}

In this study, we found that $C$. tropicalis was a robust strain with intrinsic tolerance to the pretreatment inhibitor furfural. The $\mathrm{IC}_{50}$ for furfural with xylose as the sole carbon source was $3.69 \mathrm{~g} / \mathrm{L}$, which was higher than most wild-type microbes reported in the literature to our knowledge. Mechanisms of furfural detoxification and metabolic responses in the main pathways were revealed by multiple analyses. Alcohol dehydrogenase 1 was confirmed responsible for furfural detoxification. Furfural triggered the induction of $c t A D H 1$ to degrade furfural. After furfural depletion, the transcription of $c t A D H 1$ was repressed by ethanol to maintain redox balance (Fig. 7). The regulation system gives $C$. tropicalis comparable tolerance to furfural. Understanding furfural tolerance in $C$. tropicalis at molecular level is of significant importance 


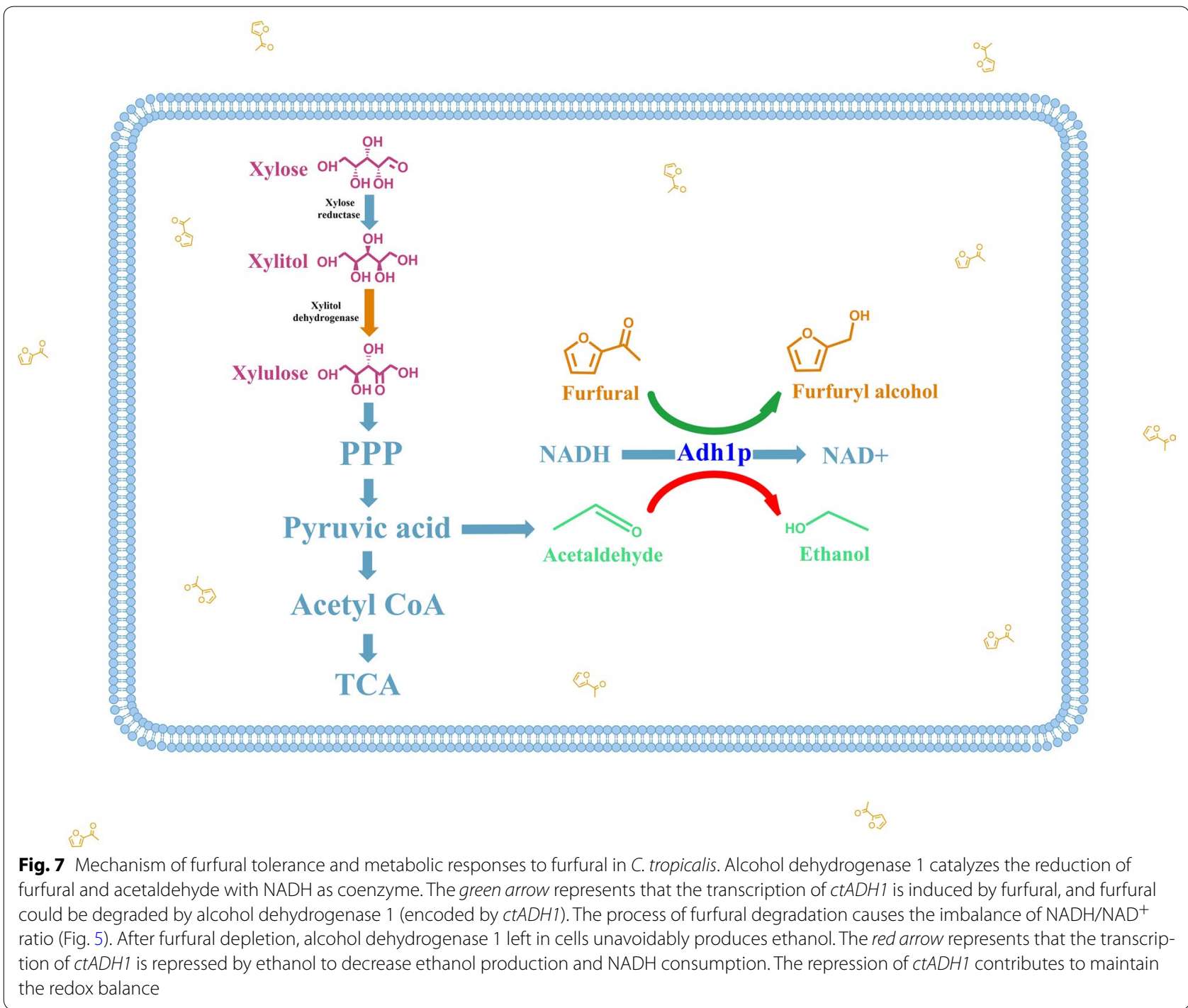

for biofuel fermentation. The study provides valuable insights into tolerance enhancement and strain modification for efficient lignocellulose fermentation.

\section{Additional file}

Additional file 1: Table S1. Strains and plasmids used in this study. Table S2. Primers used in this study. Figure S1. Furfural tolerance test evaluated by methylene blue staining. Furfural of $1,3,5,7$, and $9 \mathrm{~g} / \mathrm{L}$ was added into the culture at mid-exponential phase. After having been stained by Methylene blue solvent $30 \mathrm{~min}$, cells were observed and photoed by optical microscope. Figure S2. PCR confirmation of the specific integration in sequential ctADH1 disruption. Lane M, DNA makers; Lane A, PCR from C. tropicalis $Y E$ genome with primers $A D H 1-F$ and $A D H 1-R$ resulting in the band of $1.2 \mathrm{~kb}$ (ADH1); Lane B, PCR from C. tropicalis $Y 1$ genome with primers $A D H 1-F$ and $A D H 1-R$ resulting in the band of 1.2 and $4.3 \mathrm{~kb}$ (ADH1 and ADH1a-HisG-URA3-HisG-ADH1 b); Lane C, PCR from C. tropicalis Y2 genome with primers $A D H 1-F$ and $A D H 1-R$ resulting in the band of 1.2 and $1.7 \mathrm{~kb}$ (ADH1 and ADH1a-HisG-ADH1 b); Lane D, PCR from C. tropicalis
Y4 genome with primers $A D H 1-F$ and $A D H 1-R$ resulting in the band of 1.7 and $2.9 \mathrm{~kb}$ (ADH1a-HisG-ADH1 b and ADH1-URA3). Figure S3. Sensitivity experiment of $C$. tropicalis T4, T3, T2, and YE (parent strain). Cells of $C$. tropicalis $\mathrm{T} 4, \mathrm{~T} 3, \mathrm{~T} 2$, and YE were inoculated into $5 \mathrm{ml} \mathrm{YPD}$ medium containing $3 \mathrm{~g} / \mathrm{L}$ furfural. The cultures were incubated for $10 \mathrm{~h}$ at $30^{\circ} \mathrm{C}$. Figure $\mathbf{S 4}$. SDS-PAGE of alcohol dehydrogenase 1 expressed in E. coli. Lane M, protein molecular weight markers (Thermo Scientific, \#26610, USA); Lane A, E. coli PCA cells after IPTG induction; Lane B, E. coli PCA cells before IPTG induction; Lane C, E. coli PC cells without IPTG induction; Lane D, E. coli PEA cells after IPTG induction; Lane E, E. coli PEA cells before IPTG induction; Lane F, E. coli PE cells without IPTG induction. Molecular weight of alcohol dehydrogenase 1 is around $43 \mathrm{kDa}$. Figure S5. In vivo furfural degradation in recombined E. coli. (A) Furfural degradation of E. coli PCA (with pCS-ADH1) and PC (with PCS-27) in M9 medium; (B) Furfural degradation of E. coli PEA (with pET-ADH1) and PE (with pETDuet-1) in M9 medium.

\section{Abbreviations}

$I_{50}$ : half maximal inhibitory concentration; IPTG: $\beta$-D-1-thiogalactopyranoside; qRT-PCR: quantitative real-time PCR; YPD: yeast extract peptone dextrose; 
YNB-5FOA: yeast nitrogen base without amino acids-5-fluoroorotic acid; LB: Luria-Bertani; M9 medium: minimal basal salts medium; OD: optical density; HPLC: high-performance liquid chromatography; PPP: pentose phosphate pathway.

\section{Authors' contributions}

SW, XS, and QY designed the research. SW, ZH, RL, and LL performed the research. ZH performed the bioinformatics analysis. SW, GC, and CJ analyzed the results and wrote the manuscript. All authors read and approved the final manuscript.

\section{Author details}

${ }^{1}$ State Key Laboratory of Chemical Resource Engineering, College of Life Science and Technology, Beijing University of Chemical Technology, West Room 314, Science and Technology Building, No. 15 North Third Ring East Road, Chaoyang District, Beijing 100029, People's Republic of China. ${ }^{2}$ Deconstruction Division, Joint BioEnergy Institute, Emeryville, CA 94608, USA.

\section{Acknowledgements}

We thank Prof. Jiangye Chen for generous gift of plasmid pCUB6. We thank Mr. Xuecheng Long for help in qRT-PCR analysis.

\section{Competing interests}

The authors declare that they have no competing interests.

\section{Consent for publication}

All the authors consented on the publication of this work.

\section{Funding}

This study was supported by the National High-Tech Research and Development Program of China (2011AA02A207, 2014AA021906, and 2014AA021903) and National Natural Science Foundation of China (21376017, 21176018, 21636001, 21376017, and 21406010).

\section{Received: 11 October 2016 Accepted: 15 November 2016} Published online: 18 November 2016

\section{References}

1. Mosier N, Wyman C, Dale B, Elander R, Lee Y, Holtzapple M, et al. Features of promising technologies for pretreatment of lignocellulosic biomass. Bioresour Technol. 2005;96(6):673-86.

2. Fan X, Li M, Zhang J, Tang P, Yuan Q. Optimization of $\mathrm{SO}_{2}$-catalyzed hydrolysis of corncob for xylose and xylitol production. J Chem Technol Biotechnol. 2014;89(11):1720-6.

3. Zhang HJ, Fan XG, Qiu XL, Zhang QX, Wang WY, Li SX, et al. A novel cleaning process for industrial production of xylose in pilot scale from corncob by using screw-steam-explosive extruder. Bioprocess Biosyst Eng. 2014;37(12):2425-36.

4. Fan X, Cheng G, Zhang H, Li M, Wang S, Yuan Q. Effects of acid impregnated steam explosion process on xylose recovery and enzymatic conversion of cellulose in corncob. Carbohydr Polym. 2014;114:21-6.

5. Wang L, Yang M, Fan X, Zhu X, Xu T, Yuan Q. An environmentally friendly and efficient method for xylitol bioconversion with high-temperaturesteaming corncob hydrolysate by adapted Candida tropicalis. Process Biochem. 2011;46(8):1619-26.

6. Rasmussen H, Sørensen HR, Meyer AS. Formation of degradation compounds from lignocellulosic biomass in the biorefinery: sugar reaction mechanisms. Carbohydr Res. 2014;385:45-57.

7. Palmqvist E, Hahn-Hägerdal B. Fermentation of lignocellulosic hydrolysates. Il: inhibitors and mechanisms of inhibition. Bioresour Technol. 2000;74(1):25-33.

8. Jönsson $\sqcup$, Alriksson B, Nilvebrant N-O. Bioconversion of lignocellulose: inhibitors and detoxification. Biotechnol Biofuels. 2013;6(1):1.

9. Taherzadeh M, Gustafsson L, Niklasson C, Lidén G. Physiological effects of 5-hydroxymethylfurfural on Saccharomyces cerevisiae. Appl Microbiol Biotechnol. 2000;53(6):701-8.

10. Almarsdottir AR, Sigurbjornsdottir MA, Orlygsson J. Effect of various factors on ethanol yields from lignocellulosic biomass by Thermoanaerobacterium AK17. Biotechnol Bioeng. 2012;109(3):686-94.
11. Zaldivar J, Martinez A, Ingram LO. Effect of selected aldehydes on the growth and fermentation of ethanologenic Escherichia coli. Biotechnol Bioeng. 1999;65(1):24-33.

12. Zaldivar J, Martinez A, Ingram LO. Effect of alcohol compounds found in hemicellulose hydrolysate on the growth and fermentation of ethanologenic Escherichia coli. Biotechnol Bioeng. 2000;68(5):524-30.

13. Allen SA, Clark W, McCaffery J, Cai Z, Lanctot A, Slininger PJ, et al. Furfural induces reactive oxygen species accumulation and cellular damage in Saccharomyces cerevisiae. Biotechnol Biofuels. 2010;3(1):1.

14. Wang L, Tang P, Fan X, Yuan Q. Effect of selected aldehydes found in the corncob hemicellulose hydrolysate on the growth and xylitol fermentation of Candida tropicalis. Biotechnol Prog. 2013;29(5):1181-9.

15. Chen Y, Sheng J, Jiang T, Stevens J, Feng X, Wei N. Transcriptional profiling reveals molecular basis and novel genetic targets for improved resistance to multiple fermentation inhibitors in Saccharomyces cerevisiae. Biotechnol Biofuels. 2016;9(1):1.

16. Miller EN, Jarboe LR, Yomano L, York S, Shanmugam K, Ingram L. Silencing of NADPH-dependent oxidoreductase genes (yqhD and $\mathrm{dkgA}$ ) in furfural-resistant ethanologenic Escherichia coli. Appl Environ Microbiol. 2009;75(13):4315-23.

17. Miller EN, Jarboe LR, Turner PC, Pharkya P, Yomano LP, York SW, et al. Furfural inhibits growth by limiting sulfur assimilation in ethanologenic Escherichia coli strain LY180. Appl Environ Microbiol. 2009;75(19):6132-41.

18. Wang $X$, Miller E, Yomano L, Zhang $X$, Shanmugam $K$, Ingram L. Increased furfural tolerance due to overexpression of $\mathrm{NADH}$-dependent oxidoreductase FucO in Escherichia coli strains engineered for the production of ethanol and lactate. Appl Environ Microbiol. 2011;77(15):5132-40.

19. Lin FM, Qiao B, Yuan YJ. Comparative proteomic analysis of tolerance and adaptation of ethanologenic Saccharomyces cerevisiae to furfural, a lignocellulosic inhibitory compound. Appl Environ Microbiol. 2009;75(11):3765-76

20. Sasano Y, Watanabe D, Ukibe K, Inai T, Ohtsu I, Shimoi H, et al. Overexpression of the yeast transcription activator $\mathrm{Msn}_{2}$ confers furfural resistance and increases the initial fermentation rate in ethanol production. J Biosci Bioeng. 2012;113(4):451-5.

21. Kim D, Hahn JS. Roles of the Yap1 transcription factor and antioxidants in Saccharomyces cerevisiae's tolerance to furfural and 5-hydroxymethylfurfural, which function as thiol-reactive electrophiles generating oxidative stress. Appl Environ Microbiol. 2013;79(16):5069-77.

22. Gorsich S, Dien B, Nichols N, Slininger P, Liu Z, Skory C. Tolerance to furfural-induced stress is associated with pentose phosphate pathway genes ZWF1, GND1, RPE1, and TKL1 in Saccharomyces cerevisiae. Appl Microbiol Biotechnol. 2006;71(3):339-49.

23. Ran H, Zhang J, Gao Q, Lin Z, Bao J. Analysis of biodegradation performance of furfural and 5-hydroxymethylfurfural by Amorphotheca resinae ZN1. Biotechnol Biofuels. 2014;7(1):1.

24. Ishii J, Yoshimura K, Hasunuma T, Kondo A. Reduction of furan derivatives by overexpressing NADH-dependent Adh1 improves ethanol fermentation using xylose as sole carbon source with Saccharomyces cerevisiae harboring XR-XDH pathway. Appl Microbiol Biotechnol. 2013;97(6):2597-607.

25. Laadan B, Almeida JR, Rådström P, Hahn-Hägerdal B, Gorwa-Grauslund M. Identification of an NADH-dependent 5-hydroxymethylfurfuralreducing alcohol dehydrogenase in Saccharomyces cerevisiae. Yeast. 2008;25(3):191-8

26. Hasunuma T, Ismail KSK, Nambu Y, Kondo A. Co-expression of TAL1 and $\mathrm{ADH} 1$ in recombinant xylose-fermenting Saccharomyces cerevisiae improves ethanol production from lignocellulosic hydrolysates in the presence of furfural. J Biosci Bioeng. 2014;117(2):165-9.

27. Guigo N, Mija A, Vincent L, Sbirrazzuoli N. Chemorheological analysis and model-free kinetics of acid catalysed furfuryl alcohol polymerization. Phys Chem Chem Phys. 2007;9(39):5359-66.

28. Bozell JJ, Moens L, Elliott D, Wang Y, Neuenscwander G, Fitzpatrick S, et al. Production of levulinic acid and use as a platform chemical for derived products. Resour Conserv Recycl. 2000;28(3):227-39.

29. Wang S, Li H, Fan X, Zhang J, Tang P, Yuan Q. Metabolic responses in Candida tropicalis to complex inhibitors during xylitol bioconversion. Fungal Genet Biol. 2015;82:1-8.

30. Wang S, He Z, Yuan Q. Xylose enhances furfural tolerance in Candida tropicalis by improving NADH recycle. Chem Eng Sci. 2017;158:37-40. 
31. Ko BS, Kim J, Kim JH. Production of xylitol from D-xylose by a xylitol dehydrogenase gene-disrupted mutant of Candida tropicalis. Appl Environ Microbiol. 2006;72(6):4207-13.

32. Jeon WY, Yoon BH, Ko BS, Shim WY, Kim JH. Xylitol production is increased by expression of codon-optimized Neurospora crassa xylose reductase gene in Candida tropicalis. Bioprocess Biosyst Eng. 2012;35(1-2):191-8.

33. Mao X, Cao F, Nie X, Liu H, Chen J. The Swi/Snf chromatin remodeling complex is essential for hyphal development in Candida albicans. FEBS Lett. 2006;580(11):2615-22.

34. Haas L, Cregg J, Gleeson M. Development of an integrative DNA transformation system for the yeast Candida tropicalis. J Bacteriol. 1990;172(8):4571-7.

35. Chen Z, Li Y, Feng Y, Chen L, Yuan Q. Enzyme activity enhancement of chondroitinase $\mathrm{ABC}$ I from Proteus vulgaris by site-directed mutagenesis. RSC Adv. 2015;5(93):76040-7.

36. Delgenes J, Moletta R, Navarro J. Effects of lignocellulose degradation products on ethanol fermentations of glucose and xylose by Saccharomyces cerevisiae, Zymomonas mobilis, Pichia stipitis, and Candida shehatae. Enzyme Microb Technol. 1996;19(3):220-5.

37. Banerjee N, Bhatnagar R, Viswanathan L. Inhibition of glycolysis by furfural in Saccharomyces cerevisiae. Eur J Appl Microbiol Biotechnol. 1981;11(4):226-8.

38. Siqueira MR, Reginatto $\mathrm{V}$. Inhibition of fermentative $\mathrm{H} 2$ production by hydrolysis byproducts of lignocellulosic substrates. Renew Energy. 2015;80:109-16.

39. Bischoff KM, Liu S, Hughes SR, Rich JO. Fermentation of corn fiber hydrolysate to lactic acid by the moderate thermophile Bacillus coagulans. Biotechnol Lett. 2010;32(6):823-8.
40. De Vrije T, Bakker RR, Budde MA, Lai MH, Mars AE, Claassen PA. Efficient hydrogen production from the lignocellulosic energy crop Miscanthus by the extreme thermophilic bacteria Caldicellulosiruptor saccharolyticus and Thermotoga neapolitana. Biotechnol Biofuels. 2009;2(1):1.

41. Clarkson SM, Hamilton-Brehm SD, Giannone RJ, Engle NL, Tschaplinski $\mathrm{TJ}$, Hettich RL, et al. A comparative multidimensional LC-MS proteomic analysis reveals mechanisms for furan aldehyde detoxification in Thermoanaerobacter pseudethanolicus 39E. Biotechnol Biofuels. 2014;7(1):1.

42. Rizzi M, Erlemann P, Bui-Thanh N-A, Dellweg H. Xylose fermentation by yeasts. Appl Microbiol Biotechnol. 1988;29(2-3):148-54.

43. Rizzi M, Harwart K, Erlemann P, Bui-Thanh N-A, Dellweg H. Purification and properties of the NAD+-xylitol-dehydrogenase from the yeast Pichia stipitis. J Ferment Bioeng. 1989;67(1):20-4.

44. Seo H-M, Jeon J-M, Lee JH, Song H-S, Joo H-B, Park S-H, et al. Combinatorial application of two aldehyde oxidoreductases on isobutanol production in the presence of furfural. J Ind Microbiol Biotechnol. 2016;43(1):37-44.

45. Ask M, Bettiga M, Duraiswamy VR, Olsson L. Pulsed addition of HMF and furfural to batch-grown xylose-utilizing Saccharomyces cerevisiae results in different physiological responses in glucose and xylose consumption phase. Biotechnol Biofuels. 2013;6(1):1.

46. Palmqvist E, Almeida JS, Hahn-Hägerdal B. Influence of furfural on anaerobic glycolytic kinetics of Saccharomyces cerevisiae in batch culture. Biotechnol Bioeng. 1999;62(4):447-54.

47. Atsumi S, Hanai T, Liao JC. Non-fermentative pathways for synthesis of branched-chain higher alcohols as biofuels. Nature. 2008;451(7174):86-9.

\section{Submit your next manuscript to BioMed Central and we will help you at every step:}

- We accept pre-submission inquiries

- Our selector tool helps you to find the most relevant journal

- We provide round the clock customer support

- Convenient online submission

- Thorough peer review

- Inclusion in PubMed and all major indexing services

- Maximum visibility for your research

Submit your manuscript at www.biomedcentral.com/submit
() Biomed Central 